OPEN ACCESS

Edited by:

Giulia Rastrelli,

University of Florence, Italy

Reviewed by: Arcangelo Barbonetti,

University of L'Aquila, Italy

Giacomo Ciocca,

Sapienza University of Rome, Italy

${ }^{*}$ Correspondence:

Daye Jiang

86741852@qq.com

Specialty section

This article was submitted to Applied Neuroimaging, a section of the journal

Frontiers in Neurology

Received: 29 October 2021

Accepted: 20 January 2022

Published: 22 February 2022

Citation:

Zhang T, Yuan P, Cui Y, Yuan $W$ and

Jiang D (2022) Convergent and

Divergent Structural Connectivity of Brain White Matter Network Between

Patients With Erectile Dysfunction and

Premature Ejaculation: A Graph

Theory Analysis Study.

Front. Neurol. 13:804207.

doi: 10.3389/fneur.2022.804207

\section{Convergent and Divergent Structural Connectivity of Brain White Matter Network Between Patients With Erectile Dysfunction and Premature Ejaculation: A Graph Theory Analysis Study}

\author{
Tielong Zhang ${ }^{1}$, Peng Yuan ${ }^{2}$, Yonghua Cui ${ }^{3}$, Weibiao Yuan ${ }^{4}$ and Daye Jiang ${ }^{1 *}$ \\ ${ }^{1}$ Department of Urology, The Affiliated Jianhu Hospital of Nantong University, Jianhu People's Hospital, Yancheng, China, \\ ${ }^{2}$ Department of Intervention, The Affiliated Jianhu Hospital of Nantong University, Jianhu People's Hospital, Yancheng, China, \\ ${ }^{3}$ Department of Neurosurgery, The Affiliated Jianhu Hospital of Nantong University, Jianhu People's Hospital, Yancheng, \\ China, ${ }^{4}$ Department of Radiology, The Affiliated Jianhu Hospital of Nantong University, Jianhu People's Hospital, Yancheng, \\ China
}

Background: Sexual dysfunction, namely, erectile dysfunction (ED) and premature ejaculation (PE), has been found to be associated with abnormal structural connectivity in the brain. Previous studies have mainly focused on a single disorder, however, convergent and divergent structural connectivity patterns of the brain network between ED and PE remain poorly understood.

Methods: T1-weighted structural data and diffusion tensor imaging data of 28 patients with psychological ED, 28 patients with lifelong PE (LPE), and 28 healthy controls (HCs) were obtained to map the white matter (WM) brain networks. Then, the graph-theoretical method was applied to investigate the differences of network properties (small-world measures) of the WM network between patients with ED and LPE. Furthermore, nodal segregative and integrative parameters (nodal clustering coefficient and characteristic path length) were also explored between these patients.

Results: Small-world architecture of the brain networks were identified for both psychological ED and LPE groups. However, patients with ED exhibited increased average characteristic path length of the brain network when compared with patients with LPE and HCs. No significant difference was found in the average characteristic path length between patients with LPE and HCs. Moreover, increased nodal characteristic path length was found in the right middle frontal gyrus (orbital part) of patients with ED and LPE when compared with HCs. In addition, patients with ED had increased nodal characteristic path length in the right middle frontal gyrus (orbital part) when compared with patients with LPE.

Conclusion: Together, our results demonstrated that decreased integration of the right middle frontal gyrus (orbital part) might be a convergent neuropathological basis for 
both psychological ED and LPE. In addition, patients with ED also exhibited decreased integration in the whole WM brain network, which was not found in patients with LPE. Therefore, altered integration of the whole brain network might be the divergent structural connectivity patterns for psychological ED and LPE.

Keywords: erectile dysfunction, premature ejaculation, diffusion tensor imaging, graph theory analysis, structural connectivity

\section{INTRODUCTION}

Male sexual behavior is divided into five stages: sexual desire, sexual arousal/erection, sexual intercourse, ejaculation, and orgasm $(1,2)$. In humans, penile erection and ejaculation can occur during masturbation, copulation, or sleep, even in some non-sexually relevant context $(3,4)$. It is possible that certain brain regions may contribute to the occurrence of erection and ejaculation in different contexts (5). Previous studies have demonstrated that erection and ejaculation are considered two distinct phases of male sexual behavior, which are controlled by the peripheral and central nervous systems (6-9). Erectile dysfunction (ED) and premature ejaculation (PE) are the two most common sexual dysfunctions with many little-known links, which have a negative impact on the physical and psychosocial health and quality of sexual intercourse for both men and their female partners $(10,11)$. Approximately, 5-20\% of men suffer from moderate-to-severe ED including psychological ED (12) and $20-30 \%$ of men report experiencing PE including lifelong PE (LPE) at some point in their lives $(13,14)$. However, the diagnosis of both the psychological ED and LPE is usually based on medical and sexual history and validated questionnaires assessing the level of ED and PE (15).

The links between certain brain areas and erection have been indirectly evidenced by the proerectile effects of apomorphine in the treatment of some patients suffering from cerebral dopamine deficiency $(16,17)$. Furthermore, more attention was paid to understanding how brain regions modulate the process of ejaculation with the use of selective serotonin reuptake inhibitors in delaying ejaculation (18). The central neuropathological mechanisms of male sexual dysfunction recently received significant attention with the development of MRI, a non-invasive imaging technique, for displaying the structure and function of the brain $(5,19)$. Diffusion tensor imaging (DTI), one of the most used neuroimaging methods, can identify structural connections and explore changes in the white matter (WM) microstructure in vivo (20). Graph theory, a mathematical analysis method, quantifies the whole brain as a graph consisting of nodes linked by edges $(21,22)$. Graph analysis has revealed that the human brain network exhibits small-world network patterns, which have a balance between high segregation (measured by clustering coefficient) and integration (measured by characteristic path length) $(23,24)$. The function of the prefrontal cortex in emotion, cognition, and motivation has been mostly studied in domains other than sexual behavior (25). The role of the prefrontal cortex, especially the lateral prefrontal cortex, may be associated with the sexual arousal in response to sexual stimuli and may be involved in processing the sexual character of stimuli $(26,27)$. In addition, the activation of the orbitofrontal cortex, one component of the central inhibitory network, may be involved in the central control of ejaculation (28). Both patients with psychological ED (29) and LPE (30) showed impaired functional and structural connectivity in the brain in previous MRI studies. The altered brain regions were mainly located in the frontal cortex including the orbital prefrontal region and subcortical areas, especially the amygdala. However, convergent and divergent brain mechanisms underlying psychological ED and LPE remain poorly understood.

In this study, we used DTI data and the graph theory approach to investigate the convergent and divergent structural connectivity patterns of the brain network between patients with psychological ED and LPE. Based on previous studies, we hypothesized that psychological ED and LPE-related differences (mainly in the prefrontal cortex) would occur in the patterns of structural connectivity in the brain. We sought to determine whether WM networks would show (1) abnormal topological organization (small-world patterns) in patients with psychological ED and LPE; (2) similarities and differences in the patterns of structural connectivity (nodal clustering coefficient and characteristic path length of the prefrontal cortex) between these two groups.

\section{MATERIALS AND METHODS}

\section{Participants}

A total of 28 patients with psychological ED and 28 patients with LPE were recruited from the Department of Urology, the Affiliated Jianhu Hospital of Nantong University, Jianhu People's Hospital. In addition, 28 well-matched healthy controls (HCs) were recruited by advertisement. All the participants underwent medical and sexual history taking and physical examination.

Diagnostic criteria: (1) ED: patients with ED met the Diagnostic and Statistical Manual of Mental Disorders-Fifth Edition (DSM-V) (31) criteria (failure to obtain and maintain an erection sufficient for sexual activity or decreased erectile turgidity on $75 \%$ of sexual occasions and lasting for at least 6 months), (2) LPE: patients with LPE met the International Society for Sexual Medicine (ISSM) criteria (32) for LPE [(i) a short ejaculatory latency; (ii) a lack of perceived self-efficacy or control about the timing of ejaculation; and (iii) distress and interpersonal difficulty (related to the ejaculatory dysfunction)].

Evaluation tools: (1) psychological ED: scores of international index of erectile function (IIEF-5) $(33,34) \leq 21$, scores of premature ejaculation diagnostic tool (PEDT) $(35,36)$ $<11$, normal nocturnal erection and normal erection during 
masturbation reported by themselves. (2) LPE: PEDT scores $\geq$ 11 , IIEF-5 scores $>21$, and intravaginal ejaculation latency time (IELT) $<1$ min since the first experience of sexual intercourse.

The inclusion criteria for HCs were a total score of IIEF-5 $>21$, PEDT $<11$, and IELT $>3 \mathrm{~min}$. The inclusion criteria for all participants: (1) right-handed; (2) aged from 20 to 45 years old; (3) drug washout period (any treatment influences sexual function) $>2$ weeks; (4) frequency of sexual activity $>4$ times/week; (5) in a stable relationship with the same, nonpregnant, sexually active female partner for at least 6 months. Exclusion criteria for all participants were: (1) genital deformities including short frenulum evaluated by physical examination and other sexual dysfunction, such as hypoactive sexual desire disorder, anejaculation, and retrograde ejaculation; (2) head trauma, loss of consciousness, psychiatric diseases including depression, and anxiety evaluated by self-rating depression and anxiety scales/neurological diseases and other serious physical diseases, namely, hypertension, diabetes, coronary heart disease, liver and kidney diseases, tumor, etc.; (3) alcohol or drug abuse; and (4) any contraindications for MRI scanning.

Our study was approved by the Ethical Committee of the Affiliated Jianhu Hospital of Nantong University, Jianhu People's Hospital. Written informed consent was obtained from all participants before the study. The demographic and clinical information for all participants were presented in Table 1.

\section{Magnetic Resonance Imaging Data Acquisition and Processing}

Magnetic resonance imaging data were acquired using a 3.0 Tesla GE scanner (GE Company, America). T1-weighted images were acquired with the following parameters: repetition time $(\mathrm{TR})=8.5 \mathrm{~ms}$, echo time $(\mathrm{TE})=3.2 \mathrm{~ms}$, matrix size $=256 \times$ 256, and slice thickness $=1 \mathrm{~mm}$. DTI data were acquired with the following parameters: $\mathrm{TR}=8,724 \mathrm{~ms}$, $\mathrm{TE}=81.4 \mathrm{~ms}$, slice thickness $=2 \mathrm{~mm}, 64$ diffusion directions with $\mathrm{b}=1,000 \mathrm{~s} / \mathrm{mm}^{2}$, and an additional b0 image. Participants were instructed to close their eyes and stay awake during the MRI data acquisition.

TABLE 1 | Demographic and clinical data.

\begin{tabular}{lcccc}
\hline Variables & $\begin{array}{c}\text { Psychological ED } \\
(\boldsymbol{n}=\mathbf{2 8})\end{array}$ & $\begin{array}{c}\text { LPE } \\
(\boldsymbol{n}=\mathbf{2 8})\end{array}$ & $\begin{array}{c}\text { HCs } \\
(\boldsymbol{n}=\mathbf{2 8})\end{array}$ & $\boldsymbol{P}$ \\
\hline Age (years) & $32.82 \pm 2.23$ & $30.29 \pm 4.13$ & $31.39 \pm 6.72$ & 0.13 \\
Education level & $14.86 \pm 1.96$ & $14.54 \pm 2.62$ & $14.57 \pm 1.60$ & 0.40 \\
(years) & & & & \\
IIEF-5 scores & $10.86 \pm 3.66$ & $22.61 \pm 0.69$ & $22.71 \pm 0.76$ & $0.000015^{\mathrm{a}}$ \\
PEDT scores & $3.96 \pm 2.01$ & $14.64 \pm 3.53$ & $3.68 \pm 1.79$ & $<0.0001^{\mathrm{b}}$ \\
IELT (seconds) & $366.43 \pm 117.51$ & $30.68 \pm 18.56$ & $405.00 \pm 98.83$ & $<0.0001^{\mathrm{C}}$
\end{tabular}

$E D$, erectile dysfunction; $L P E$, lifelong premature ejaculation; HCs, healthy controls; IELT, intravaginal ejaculation latency time; PEDT, premature ejaculation diagnostic tool.

a Significant differences were found in psychological ED when compared with LPE and HCs.

$b, c$ Significant differences were found in LPE when compared with psychological ED and HCs. Multiple and two independent samples non-parametric tests were conducted to explore the differences of demographic and clinical data among the three groups. $P<$ 0.05 was considered as statistically significant.
MRI data processing was implemented using the FMRIB Diffusion Toolbox (FDT) in FMRIB's Software Library (37). The data processing procedure was as follows: (1) brain extraction; (2) corrections for head motion artifacts and eddy current distortions in the DTI dataset by registering the diffusionweighted images to the b0 images; (3) diffusion tensor estimation by the Stejskal and Tanner equation (38); (4) calculation of fractional anisotropy (FA) (the extent of directionality of water diffusion can be expressed as FA, which is a measure reflecting the directional organization of WM in the brain) of each voxel and FA maps describing the distribution of WM fiber tracts in the whole brain; (5) reconstruction of WM pathways using fiber assignment by continuous tracking (FACT) algorithm.

In this study, the parcellation process was performed in the native space. First, the T1-weighted images of each subject were co-registered to their corresponding b0 images in the DTI space. Second, the coregistered T1-weighted images were normalized to the International Consortium of Brain Mapping 152 T1 template in the Montreal Neurological Institute (MNI) space. Lastly, the inverse transformations were applied to warp the automated anatomical labeling template from the MNI space to the DTI native space (39). In addition, the tractography was terminated if the turned an angle $>50^{\circ}$ or FA value of a reached voxel $<0.2$.

\section{Brain Network Construction and Analysis}

The WM brain networks were constructed as described in previous studies $(40,41)$. Nodes (brain regions) and edges (structural connectivity/WM fiber tracts) are the two fundamental elements of a network (structural/WM brain network) (42). To define the nodes, the entire cerebral cortex was divided into 90 cortical and subcortical regions (45 regions in each hemisphere). To define the edges, a threshold of 3 fiber streamlines was selected and the mean FA value of the fiber connected two brain regions was defined as the weight of the edge between these regions. Finally, FAweighted $90 \times 90 \mathrm{WM}$ brain networks of all the subjects were constructed.

The small-world measures include the clustering coefficient $\left(C_{p}\right)$ (a measure of segregation; local information transformation capacity), characteristic path length $\left(L_{p}\right)$ (a measure of integration; global information transformation capacity) of the network, their corresponding normalized $C_{p}(\gamma)$ and $L_{p}(\lambda)$, and small-worldness $(\sigma)$ (the balance between local segregation and global integration) (42). These measures were calculated to evaluate the information integration and segregation of the whole brain network using the Brain Connectivity Toolbox. In addition, the nodal parameters, namely, nodal clustering coefficient $\left(C_{i}\right)$ and characteristic path length $\left(L_{i}\right)$ were calculated to explore the integration and segregation of the brain regions in the network.

\section{Statistical Analysis}

Statistical analyses were performed by IBM SPSS statistics (version 20) for Windows (IBM Corporation, Armonk, New York, USA). Multiple and two independent samples nonparametric tests were conducted to explore the differences of demographic and clinical data among the three groups $(P<$ 
0.05). Then ANOVA was employed to identify group differences in the graph metrics of both network $(P<0.05)$ and node $(P<$ 0.01 ). In addition, false discovery rate (FDR) correction (number of tests $=90$ (brain regions); $P_{i}<0.05 \times i / 90 ; P_{1} \leq P_{2} \leq$ $\left.P_{3} \ldots \leq P_{i} ; i=1,2,3 \ldots 90\right)$ was applied to account for multiple comparisons of nodal parameters calculations.

\section{RESULTS}

\section{Global Network Properties}

Small-world properties were demonstrated in both the patients and HCs $(\gamma>>1 ; \lambda \approx 1 ; \sigma>>1)$. However, patients with psychological ED, relative to patients with LPE and HCs, showed significant increases in the average characteristic path length $L_{p}$ of the brain network. No significant differences were found in the average characteristic path length $L_{p}$ of the brain network between patients with LPE and HCs. Concerning other smallworld measures $\left(C_{p} ; \gamma ; \lambda ; \sigma\right)$, no significant differences were found among the three groups (Figure 1 and Table 2).

\section{Regional Nodal Characteristics}

Among the three groups, we found that the regions with significant group effects of nodal characteristic path length $\left(L_{i}\right)$ were mainly distributed in the frontal and subcortical regions (Figure 2, Table 3). However, only the right middle frontal

TABLE 2 | Differences of small-world measures in the white matter brain networks.

\begin{tabular}{lccccc}
\hline $\begin{array}{l}\text { Network } \\
\text { metrics }\end{array}$ & $\begin{array}{c}\text { Psychological } \\
\text { ED } \\
(\boldsymbol{n}=\mathbf{2 8})\end{array}$ & $\begin{array}{c}\text { LPE } \\
(\boldsymbol{n}=\mathbf{2 8})\end{array}$ & $\begin{array}{c}\text { HCs } \\
(\boldsymbol{n}=\mathbf{2 8})\end{array}$ & $\boldsymbol{F}$ & $\boldsymbol{P}$ \\
\hline $\begin{array}{l}\text { Clustering } \\
\text { coefficient }\left(C_{p}\right)\end{array}$ & $0.13 \pm 0.019$ & $0.14 \pm 0.018$ & $0.14 \pm 0.020$ & 1.29 & 0.28 \\
$\begin{array}{l}\text { Characteristic } \\
\text { path length }\left(L_{p}\right)\end{array}$ & $9.54 \pm 1.32$ & $8.95 \pm 0.72$ & $8.85 \pm 1.05$ & 3.51 & $0.035^{\mathrm{a}}$ \\
$\begin{array}{l}\text { Normalized } \\
C_{p}(\gamma)\end{array}$ & $4.61 \pm 0.50$ & $4.59 \pm 0.61$ & $4.57 \pm 0.49$ & 0.027 & 0.97 \\
Normalized & $1.22 \pm 0.034$ & $1.21 \pm 0.062$ & $1.19 \pm 0.044$ & 1.62 & 0.20 \\
$\begin{array}{l}L_{p}(\lambda) \\
\text { Small-worldness } \\
(\sigma)\end{array}$ & $3.79 \pm 0.38$ & $3.81 \pm 0.48$ & $3.83 \pm 0.33$ & 0.079 & 0.92 \\
& & & & & \\
\end{tabular}

$E D$, erectile dysfunction; $L P E$, lifelong premature ejaculation; HCs, healthy controls. a Significant differences were found in psychological ED when compared with LPE and HCs. One-way ANOVA was performed among three groups and two-sample t-test was performed as post-hoc tests. $P<0.05$ was considered as statistically significant.
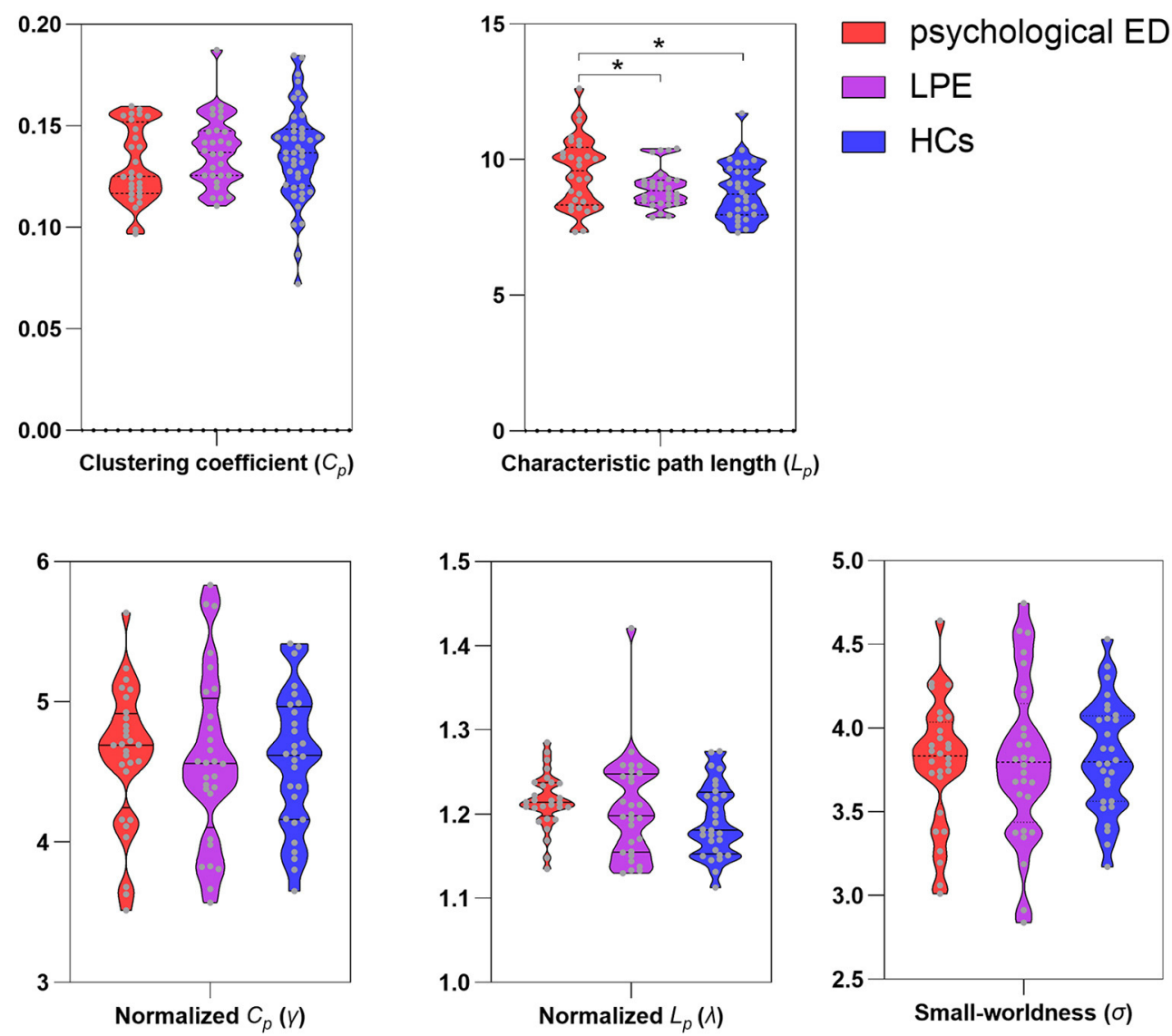

FIGURE 1 | Differences of small-world measures among three groups. ED, erectile dysfunction; LPE, lifelong premature ejaculation; HCs, healthy controls. *Significant differences between two groups detected by two-sample $t$-test. $P<0.05$ was considered as statistically significant. 

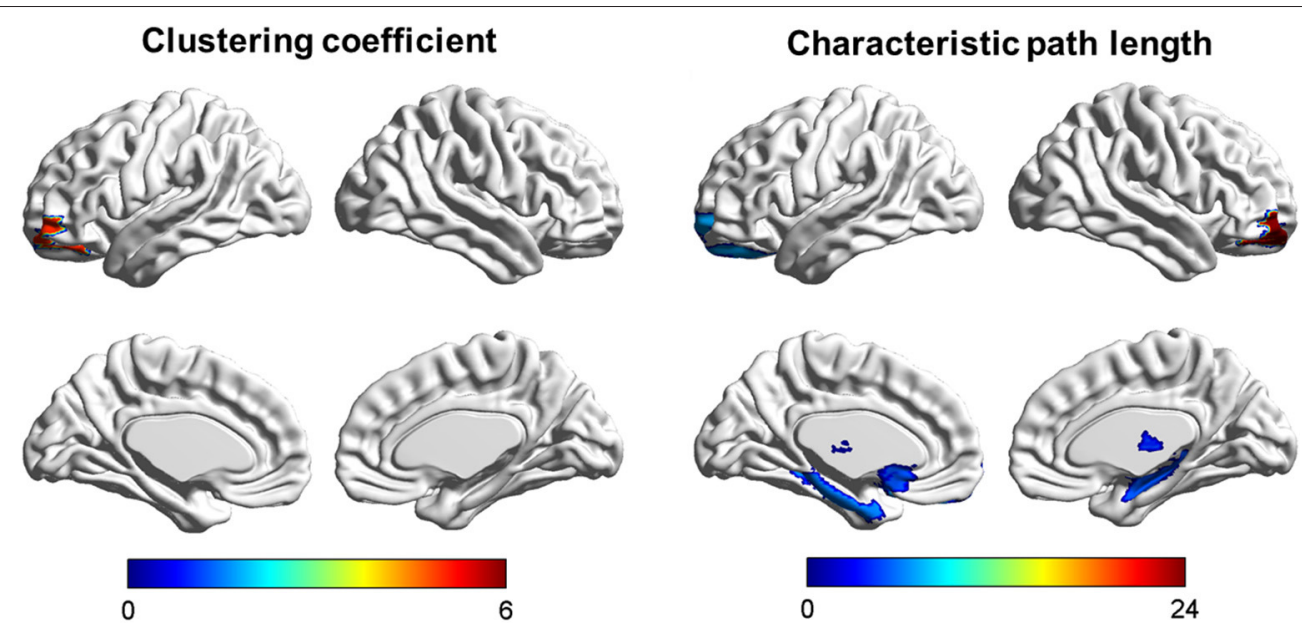

FIGURE 2 | Brain areas showing abnormal segregation and integration among the three groups. Color bar: $F$-values detected by one-way ANOVA test. $P<0.01$ was considered as statistically significant.

TABLE 3 | Differences of nodal segregative and integrative parameters in the white matter brain networks.

\begin{tabular}{cccccc}
\hline Nodal metrics & $\begin{array}{c}\text { Psychological } \\
\text { ED } \\
(n=28)\end{array}$ & $\begin{array}{c}\text { LPE } \\
(n=28)\end{array}$ & $\begin{array}{c}\text { HCs } \\
(n=28)\end{array}$ & $F$ & $P$ \\
& & & &
\end{tabular}

\begin{tabular}{|c|c|c|c|c|c|}
\hline \multicolumn{6}{|c|}{ Nodal clustering coefficient $\left(C_{i}\right)$} \\
\hline $\begin{array}{l}\text { Left middle } \\
\text { frontal gyrus } \\
\text { (orbital part) }\end{array}$ & $0.14 \pm 0.066$ & $0.19 \pm 0.077$ & $0.20 \pm 0.062$ & 5.47 & 0.0059 \\
\hline \multicolumn{6}{|c|}{ Nodal characteristic path length $\left(L_{i}\right)$} \\
\hline $\begin{array}{l}\text { Left superior } \\
\text { frontal gyrus } \\
\text { (orbital part) }\end{array}$ & $9.49 \pm 1.81$ & $8.28 \pm 1.32$ & $8.11 \pm 1.44$ & 6.67 & 0.0021 \\
\hline $\begin{array}{l}\text { Right middle } \\
\text { frontal gyrus } \\
\text { (orbital part) }\end{array}$ & $11.15 \pm 1.98$ & $9.60 \pm 0.94$ & $8.73 \pm 0.70$ & 23.73 & $<0.0001^{\mathrm{a}}$ \\
\hline $\begin{array}{l}\text { Left olfactory } \\
\text { cortex }\end{array}$ & $10.12 \pm 1.89$ & $9.14 \pm 2.52$ & $8.43 \pm 1.11$ & 5.47 & 0.0059 \\
\hline $\begin{array}{l}\text { Right } \\
\text { hippocampus }\end{array}$ & $8.89 \pm 1.92$ & $7.87 \pm 1.02$ & $7.82 \pm 1.22$ & 4.94 & 0.0094 \\
\hline $\begin{array}{l}\text { Left } \\
\text { parahippocampal } \\
\text { gyrus }\end{array}$ & $10.77 \pm 1.90$ & $10.20 \pm 1.06$ & $9.46 \pm 1.33$ & 5.63 & 0.0051 \\
\hline Left putamen & $8.01 \pm 1.11$ & $7.33 \pm 0.91$ & $7.13 \pm 1.00$ & 5.79 & 0.0044 \\
\hline Right thalamus & $8.66 \pm 1.45$ & $7.87 \pm 0.65$ & $7.78 \pm 1.03$ & 5.48 & 0.0059 \\
\hline
\end{tabular}

$E D$, erectile dysfunction; $L P E$, lifelong premature ejaculation; HCs, healthy controls. a Significant differences were found between all groups. One-way ANOVA was performed among the three groups and two-sample t-test was performed as post-hoc tests. $P<$ 0.01 was considered as statistically significant.

gyrus (orbital part) showed significant group differences after FDR correction (Figure 3, Table 3). Compared to HCs, both patients with psychological ED and LPE exhibited increased nodal characteristic path length $\left(L_{i}\right)$ in the right middle frontal gyrus (orbital part). Moreover, patients with psychological ED showed increased nodal characteristic path length in the right middle frontal gyrus (orbital part) when compared with patients with LPE.

\section{DISCUSSION}

In this study, we evaluated the architecture of WM networks in patients with ED (psychological ED characterized by normal nocturnal erection and normal erection during masturbation) and LPE, which presented similar small-world organization. However, patients with psychological ED exhibited increased characteristic path length $L_{p}$ when compared with patients with LPE. Moreover, significantly increased nodal characteristic path length $\left(L_{i}\right)$ was found in patients with psychological ED and LPE compared with HCs, mainly located in the right middle frontal gyrus (orbital part). For patients with psychological ED, a significant decline of $L_{i}$ was found in the right middle frontal gyrus (orbital part) when compared with patients with LPE. These findings improved our understanding of convergent and divergent neuropathological mechanisms in the brain WM network level between psychological ED and LPE.

Previous studies showed that ED and PE were strongly correlated with psychological factors (e.g., alexithymia) and these patients were lack of emotional awareness and shared the same difficulty in identifying and communicating emotions (i.e., alexithymia) (43-45). The brain functional and structural imaging results demonstrated that the development of alexithymia might be caused by the altered connectivity among the superior frontal gyrus, inferior temporal gyrus, anterior cingulate gyrus, and insula $(46,47)$. In addition, an increasing body of neuroimaging evidence has identified structural brain abnormalities in psychogenic and venous ED, and LPE, mainly in the prefrontal cortex, limbic system, and subcortical areas (48-51). Previous studies have revealed impaired WM in the prefrontal cortex of patients with psychological ED and LPE $(52,53)$. In addition, ED owing to psychological factors and patients with PE having depressive symptoms had altered topological characteristics in the prefrontal regions of the WM brain network $(29,54)$. Regarding the global topological organization, the small world is one of the major organizational principles of the human brain (24). We found a conserved 


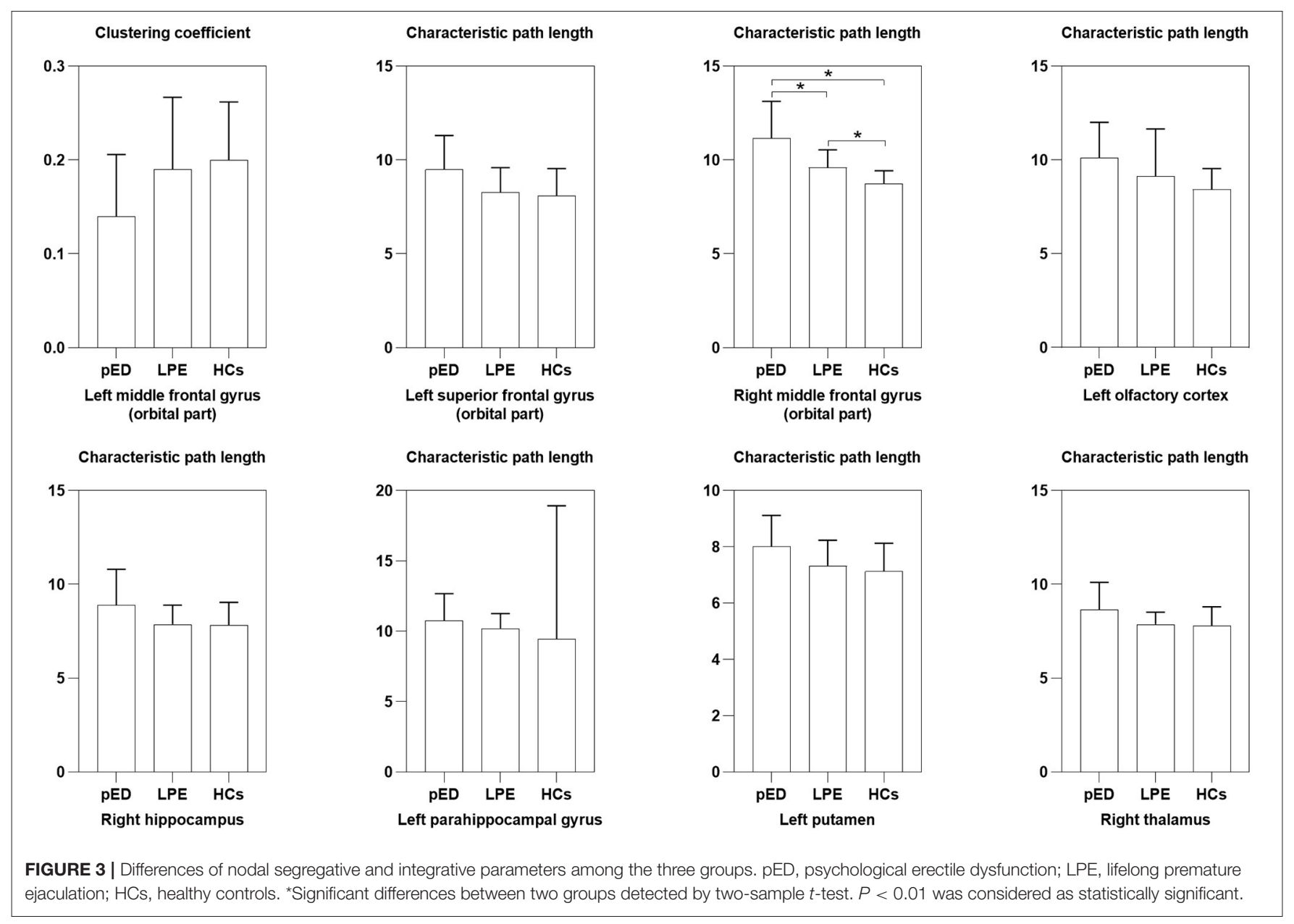

small-world architecture in both patients with psychological ED and LPE, and in HCs, which was consistent with previous studies $(52,55)$. This demonstrated that the human brain had evolved into a complex but efficient interconnected system, which was capable of parallel information processing with high efficiency at a low cost (24). However, patients with psychological ED exhibited significantly increased characteristic path length $L_{p}$ of the brain network when compared with both patients with LPE and HCs. The average characteristic path length $L_{p}$ of the brain network represents the averaged ability of information transformation between regions in the whole brain. Therefore, this finding of increased $L_{p}$ might suggest that the information transfer between brain regions became more difficult with higher wiring cost (the lower ability of the brain to globally integrate information) in patients with psychological ED.

Regarding the nodal topological properties, significant increased nodal characteristic path length $\left(L_{i}\right)$ was discovered in both patients with psychological ED and LPE, mainly located in the right middle frontal gyrus (orbital part). The measure of $L_{i}$ represents the ability of information transfer from one region to other regions in the whole brain (42). Increased $L_{i}$ reflected the disruption of brain regions' structural connection, which suggested decreased parallel information transfer between distant brain regions. The prefrontal cortex is the most complex and highly evolved neocortex region, which accepts different afferent nerve fibers from other brain areas $(56,57)$. The prefrontal cortex was thought to play a central role in the pathophysiology of male sexual dysfunctions in previous functional and structural MRI studies $(5,58,59)$.

The middle frontal gyrus has been found to be involved functionally in cognition and emotional regulation $(60,61)$. The middle frontal gyrus (orbital part), a region of the orbitofrontal cortex is implicated with the inhibitory control of the human brain $(62,63)$. Previous studies demonstrated that the process of ejaculation was associated with decreased activity in this area (64-66). The orbitofrontal cortex was thought to modulate the activity of the amygdala, which showed increased activity in patients with PE $(48,67)$. The PE-related abnormalities of function and structure were observed in the frontal regions, namely, the orbital frontal cortex, superior frontal gyrus (medial), middle frontal gyrus (orbital part), inferior frontal gyrus (orbital part), and cingulate gyrus $(49,59,64,65,68)$. These findings suggested that patients with PE had an abnormal brain control network, which might contribute to the reduced central control of rapid ejaculation (28). Therefore, decreased ability of information integration in the middle frontal gyrus (orbital part) might contribute to the reduced inhibition of ejaculatory reflex in patients with PE. The increased nodal characteristic path length 
$\left(L_{i}\right)$ of the right middle frontal gyrus (orbital part) might be involved in the psychopathology and pathophysiology of rapid ejaculation in patients with LPE.

The middle frontal gyrus is also an important part of the lateral prefrontal cortex, which has been found to be actively involved in maintaining the representation of sexual information in working memory $(5,27)$. Previous studies showed that the lateral prefrontal cortex had a critical role in male sexual arousal and sexual behavior $(27,69)$. The middle frontal gyrus (orbital part) is a core region of the central executive network in the brain, which is primarily implicated in cognitive control, response inhibition, and attention $(60,70)$. The previous study demonstrated that patients with minor changes (fewer subregions) of the lateral prefrontal cortex exhibited inhibition in behavior while patients with superior abnormalities (more subregions) of this region had both aberrant sexual inhibition and insufficient cognition and attention to sexual targets $(27,71)$. This finding illustrated that superior increased nodal characteristic path length $\left(L_{i}\right)$ of the right middle frontal gyrus (orbital part) might be an important neural pathological feature of psychological ED, which was different from PE. Therefore, we speculated that the WM brain network of psychological ED was more sensitive than that of patients with LPE and psychological ED had severely impaired integration in the brain than LPE. Both ED and PE, especially psychological ED and LPE might be related to impaired structural brain connectivity. For these patients, more targeted treatments to improve brain functional and structural connectivity should be considered.

There were several limitations in this study. First, this was a cross-sectional study, which could not find the causal relationships between abnormal measures of WM network and male sexual dysfunction. Second, the sample size of this study was relatively small and future longitudinal studies with large sample sizes were of particular interest in this regard. Third, the lack of standardized sexual evaluation was another limitation, especially the methods used to distinguish psychological sexual dysfunction and organic sexual dysfunction. Finally, patients with psychological ED included in this study were considered to be suffered from psychogenic factors, which might be also related to the altered brain connectivity. Therefore, the relationships between psychological ED and psychogenic factors should be further explored.

\section{REFERENCES}

1. Hull EM, Dominguez JM. Neuroendocrine regulation of male sexual behavior. Comprehen Physiol. (2019) 9:1383-410. doi: 10.1002/cphy.c180018

2. Jennings KJ, de Lecea L. Neural and hormonal control of sexual behavior. Endocrinology. (2020) 161:150. doi: 10.1210/endocr/bqaa150

3. Schmidt MH. Sleep-related erection neurophysiology: a journey of discovery. Sleep Med. (2018) 49:24-7. doi: 10.1016/j.sleep.2018.05.032

4. Yang CC, Jiang X. Clinical autonomic neurophysiology and the male sexual response: an overview. J Sex Med. (2009) 6(Suppl. 3):2218. doi: 10.1111/j.1743-6109.2008.01180.x

5. Cheng JC, Secondary J, Burke WH, Fedoroff JP, Dwyer RG. Neuroimaging and sexual behavior: identification of regional and functional

\section{CONCLUSION}

In conclusion, the results of this study demonstrated that the brain WM network of both patients with psychological ED and LPE had small-world organization. However, decreased global integration of the brain was found in patients with psychological $\mathrm{ED}$, which was the divergent structural connectivity patterns in the brain network level between psychological ED and LPE. Moreover, the right middle frontal gyrus (orbital part) exhibited more remarkably decreased integration in patients with psychological ED when compared with patients with LPE. This study provided another perspective for understanding the convergent and divergent neuropathological mechanisms between psychological ED and LPE.

\section{DATA AVAILABILITY STATEMENT}

The raw data supporting the conclusions of this article will be made available by the authors, without undue reservation.

\section{ETHICS STATEMENT}

The studies involving human participants were reviewed and approved by the Ethical Committee of the Affiliated Jianhu Hospital of Nantong University, Jianhu People's Hospital. The patients/participants provided their written informed consent to participate in this study.

\section{AUTHOR CONTRIBUTIONS}

TZ and DJ designed the experiments, wrote the manuscript, and approved the final manuscript. TZ, DJ, PY, and WY contributed to clinical data collection and assessment. TZ, DJ, and YC analyzed the results. All authors contributed to the article and approved the submitted version.

\section{FUNDING}

This study was supported by the grants of Yancheng Medical Technology Development Project (No. YK2015076), 333 Project of Jiangsu Province (No. BRA2017219), and Medical project of Jiangsu Health Commission (No. 233).

differences. Curr Psychiatry Rep. (2015) 17:55. doi: 10.1007/s11920-0150593-X

6. Temel Y, Hafizi S, Tan S, Visser-Vandewalle V. Role of the brain in the control of erection. Asian J Androl. (2006) 8:25964. doi: 10.1111/j.1745-7262.2006.00110.x

7. Tsujimura A, Miyagawa Y, Fujita K, Matsuoka Y, Takahashi T, Takao $\mathrm{T}$, et al. Brain processing of audiovisual sexual stimuli inducing penile erection: a positron emission tomography study. J Urol. (2006) 176:67983. doi: 10.1016/j.juro.2006.03.032

8. Facchinetti P, Giuliano F, Laurin M, Bernabé J, Clément P. Direct brain projections onto the spinal generator of ejaculation in the rat. Neuroscience. (2014) 272:207-16. doi: 10.1016/j.neuroscience.2014. 04.064 
9. Holstege G, Georgiadis JR, Paans AM, Meiners LC, van der Graaf FH, Reinders AA. Brain activation during human male ejaculation. J Neurosci. (2003) 23:9185-93. doi: 10.1523/JNEUROSCI.23-27-09185.2003

10. Ghalayini IF, Al-Ghazo MA, Al-Azab R, Bani-Hani I, Matani YS, Barham $\mathrm{AE}$, et al. Erectile dysfunction in a Mediterranean country: results of an epidemiological survey of a representative sample of men. Int J Impotence Res. (2010) 22:196-203. doi: 10.1038/ijir.2009.65

11. Verze P, Arcaniolo D, Palmieri A, Cai T, La Rocca R, Franco M, et al. Premature ejaculation among Italian men: prevalence and clinical correlates from an observational, non-interventional, cross-sectional, epidemiological study (IPER). Sex Med. (2018) 6:193-202. doi: 10.1016/j.esxm.2018.04.005

12. Feldman HA, Goldstein I, Hatzichristou DG, Krane RJ, McKinlay JB. Impotence and its medical and psychosocial correlates: results of the Massachusetts Male Aging Study. J Urol. (1994) 151:54-61. doi: 10.1016/S0022-5347(17)34871-1

13. Laumann EO, Nicolosi A, Glasser DB, Paik A, Gingell C, Moreira E, et al. Sexual problems among women and men aged 40-80 y: prevalence and correlates identified in the Global Study of Sexual Attitudes and Behaviors. Int J Impotence Res. (2005) 17:39-57. doi: 10.1038/sj.ijir.3901250

14. Porst H, Montorsi F, Rosen RC, Gaynor L, Grupe S, Alexander J. The Premature Ejaculation Prevalence and Attitudes (PEPA) survey: prevalence, comorbidities, and professional help-seeking. Eur Urol. (2007) 51:816-23; discussion: 824. doi: 10.1016/j.eururo.2006.07.004

15. Salonia A, Bettocchi C, Boeri L, Capogrosso P, Carvalho J, Cilesiz NC, et al. European association of urology guidelines on sexual and reproductive health-2021 update: male sexual dysfunction. Eur Urol. (2021) 80:33357. doi: 10.1016/j.eururo.2021.06.007

16. Sanna F, Succu S, Hübner H, Gmeiner P, Argiolas A, Melis MR. Dopamine D2like receptor agonists induce penile erection in male rats: differential role of D2, D3 and D4 receptors in the paraventricular nucleus of the hypothalamus. Behav Brain Res. (2011) 225:169-76. doi: 10.1016/j.bbr.2011.07.018

17. Yang $\mathrm{Y}$, Liu $\mathrm{H}$, Lin $\mathrm{T}$, Kuo $\mathrm{Y}$, Hsieh $\mathrm{T}$. Relationship between erectile dysfunction, comorbidity, and Parkinson's disease: evidence from a population-based longitudinal study. J Clin Neurol. (2017) 13:250-8. doi: $10.3988 /$ jen.2017.13.3.250

18. Waldinger MD, Berendsen HH, Blok BF, Olivier B, Holstege G. Premature ejaculation and serotonergic antidepressants-induced delayed ejaculation: the involvement of the serotonergic system. Behav Brain Res. (1998) 92:1118. doi: 10.1016/S0166-4328(97)00183-6

19. Yin T, Li Z, Xiong J, Lan L, Sun R, Ren F, et al. Neuroimaging biomarkers of psychogenic erectile dysfunction: protocol for a systematic review. BMJ Open. (2019) 9:e030061. doi: 10.1136/bmjopen-2019-030061

20. Atkinson-Clement C, Pinto S, Eusebio A, Coulon O. Diffusion tensor imaging in Parkinson's disease: review and meta-analysis. NeuroImage Clin. (2017) 16:98-110. doi: 10.1016/j.nicl.2017.07.011

21. Sporns O. Graph theory methods: applications in brain networks. Dial Clin Neurosci. (2018) 20:111-21. doi: 10.31887/DCNS.2018.20.2/osporns

22. Vecchio F, Miraglia F, Maria Rossini P. Connectome: graph theory application in functional brain network architecture. Clin Neurophysiol Practice. (2017) 2:206-13. doi: 10.1016/j.cnp.2017.09.003

23. Bassett DS, Bullmore ET. Small-world brain networks revisited. Neuroscientist. (2017) 23:499-516. doi: 10.1177/1073858416667720

24. Liao X, Vasilakos AV, He Y. Small-world human brain networks: perspectives and challenges. Neurosci Biobehav Rev. (2017) 77:286300. doi: 10.1016/j.neubiorev.2017.03.018

25. Hiser J, Koenigs M. The multifaceted role of the ventromedial prefrontal cortex in emotion, decision making, social cognition, and psychopathology. Biol Psychiatry. (2018) 83:638-47. doi: 10.1016/j.biopsych.2017.10.030

26. Stoléru S, Fonteille V, Cornélis C, Joyal C, Moulier V. Functional neuroimaging studies of sexual arousal and orgasm in healthy men and women: a review and meta-analysis. Neurosci Biobehav Rev. (2012) 36:1481509. doi: 10.1016/j.neubiorev.2012.03.006

27. Spinella M. The role of prefrontal systems in sexual behavior. Int J Neurosci. (2007) 117:369-85. doi: 10.1080/00207450600588980

28. Yang X, Gao M, Zhang L, Liu L, Liu P, Sun J, et al. Central neural correlates during inhibitory control in lifelong premature ejaculation patients. Front Hum Neurosci. (2018) 12:206. doi: 10.3389/fnhum.2018.00206
29. Chen J, Huang X, Liu S, Lu C, Dai Y, Yao Z, et al. Disrupted topological properties of brain networks in erectile dysfunction patients owing predominantly to psychological factors: a structural and functional neuroimaging study. Andrology. (2020) 8:381-91. doi: 10.1111/andr.12684

30. Gao S, Chen J, Xu Y, Liu S, Lu C, Guan Y, et al. Altered structural and functional connectivity contribute to rapid ejaculation: insights from a multimodal neuroimaging study. Neuroscience. (2021) 471:93101. doi: 10.1016/j.neuroscience.2021.06.034

31. Segraves RT. Considerations for diagnostic criteria for erectile dysfunction in DSM V. J Sex Med. (2010) 7(2 Pt 1):65460. doi: 10.1111/j.1743-6109.2009.01684.x

32. Althof SE, Abdo CH, Dean J, Hackett G, McCabe M, McMahon CG, et al. International society for sexual medicine's guidelines for the diagnosis and treatment of premature ejaculation. J Sex Med. (2010) 7:294769. doi: $10.1111 /$ j.1743-6109.2010.01975.x

33. Rosen RC, Riley A, Wagner G, Osterloh IH, Kirkpatrick J, Mishra A. The international index of erectile function (IIEF): a multidimensional scale for assessment of erectile dysfunction. Urology. (1997) 49:82230. doi: 10.1016/S0090-4295(97)00238-0

34. Tang DD, Li C, Peng DW, Zhang XS. Validity of premature ejaculation diagnostic tool and its association with International Index of Erectile Function-15 in Chinese men with evidence-based-defined premature ejaculation. Asian J Androl. (2018) 20:19-23. doi: 10.4103/aja.aja_9_17

35. Kam SC, Han DH, Lee SW. The diagnostic value of the premature ejaculation diagnostic tool and its association with intravaginal ejaculatory latency time. $J$ Sex Med. (2011) 8:865-71. doi: 10.1111/j.1743-6109.2010.02151.x

36. Huang YP, Chen B, Ping P, Wang HX, Hu K, Zhang T, et al. The premature ejaculation diagnostic tool (PEDT): linguistic validity of the Chinese version. J Sex Med. (2014) 11:2232-8. doi: 10.1111/jsm.12612

37. Jenkinson M, Beckmann CF, Behrens TE, Woolrich MW, Smith SM. FSL. NeuroImage. (2012) 62:782-90. doi: 10.1016/j.neuroimage.2011.09.015

38. Stejskal E. Spin diffusion measurements : spin echoes in the presence of a timedependent field gradient. J Chem Phys. (1965) 42:288. doi: 10.1063/1.1695690

39. Tzourio-Mazoyer N, Landeau B, Papathanassiou D, Crivello F, Etard $\mathrm{O}$, Delcroix N, et al. Automated anatomical labeling of activations in SPM using a macroscopic anatomical parcellation of the MNI MRI single-subject brain. NeuroImage. (2002) 15:273-89. doi: 10.1006/nimg.20 01.0978

40. Zhang Y, Li M, Wang R, Bi Y, Li Y, Yi Z, et al. Abnormal brain white matter network in young smokers: a graph theory analysis study. Brain Imaging Behav. (2018) 12:345-56. doi: 10.1007/s11682-017-9699-6

41. Zhou C, Ping L, Chen W, He M, Xu J, Shen Z, et al. Altered white matter structural networks in drug-naïve patients with obsessive-compulsive disorder. Brain Imaging Behav. (2021) 15:700-10. doi: 10.1007/s11682-020-00278-7

42. Rubinov $M$, Sporns $O$. Complex network measures of brain connectivity: uses and interpretations. NeuroImage. (2010) 52:1059-69. doi: 10.1016/j.neuroimage.2009.10.003

43. Michetti PM, Rossi R, Bonanno D, Tiesi A, Simonelli C. Male sexuality and regulation of emotions: a study on the association between alexithymia and erectile dysfunction (ED). Int J Impotence Res. (2006) 18:1704. doi: $10.1038 /$ sj.ijir.3901386

44. Michetti PM, Rossi R, Bonanno D, De Dominicis C, Iori F, Simonelli C. Dysregulation of emotions and premature ejaculation (PE): alexithymia in 100 outpatients. J Sex Med. (2007) 4:14627. doi: 10.1111/j.1743-6109.2007.00564.x

45. Madioni F, Mammana LA. Toronto alexithymia scale in outpatients with sexual disorders. Psychopathology. (2001) 34:95-8. doi: 10.1159/000049287

46. Fang Y, Li M, Mei M, Sun X, Han D. Characteristics of brain functional and structural connectivity in alexithymic students. Neuropsychiatr Dis Treat. (2018) 14:2609-15. doi: 10.2147/NDT.S174015

47. Han D, Li M, Mei M, Sun X. The functional and structural characteristics of the emotion network in alexithymia. Neuropsychiatr Dis Treat. (2018) 14:991-8. doi: 10.2147/NDT.S154601

48. Geng B, Gao M, Wu J, Yang G, Liu C, Piao R, et al. Smaller volume and altered functional connectivity of the amygdala in patients with lifelong premature ejaculation. Eur Radiol. (2021) 31:8429-37. doi: 10.1007/s00330-021-08002-9 
49. Atalay HA, Sonkaya AR, Ozbir S, Culha MG, Degirmentepe B, Bayraktarli R, et al. Are there differences in brain morphology in patients with lifelong premature ejaculation? J Sex Med. (2019) 16:992-8. doi: 10.1016/j.jsxm.2019.04.008

50. Li L, Fan W, Li J, Li Q, Wang J, Fan Y, et al. Abnormal brain structure as a potential biomarker for venous erectile dysfunction: evidence from multimodal MRI and machine learning. Eur Radiol. (2018) 28:3789800. doi: 10.1007/s00330-018-5365-7

51. Wang Y, Dong M, Guan M, Wu J, He Z, Zou Z, et al. Aberrant insulacentered functional connectivity in psychogenic erectile dysfunction patients: a resting-state fMRI study. Front Hum Neurosci. (2017) 11:221. doi: 10.3389/fnhum.2017.00221

52. Chen J, Chen Y, Gao Q, Chen G, Dai Y, Yao Z, et al. Brain structural network topological alterations of the left prefrontal and limbic cortex in psychogenic erectile dysfunction. Int J Neurosci. (2018) 128:393403. doi: 10.1080/00207454.2017.1387116

53. Gao M, Yang X, Liu L, Fei N, Xi Y, Guo F, et al. Abnormal white matter microstructure in lifelong premature ejaculation patients identified by tract-based spatial statistical analysis. J Sex Med. (2018) 15:12729. doi: 10.1016/j.jsxm.2018.07.012

54. Chen J, Yang J, Xiang Z, Huang X, Lu C, Liu S, et al. Graph theory analysis reveals premature ejaculation is a brain disorder with altered structural connectivity and depressive symptom: a DTI-based connectome study. Eur J Neurosci. (2021) 53:1905-21. doi: 10.1111/ejn.15048

55. Chen J, Chen Y, Chen G, Dai Y, Yao Z, Lu Q. Altered brain networks in psychogenic erectile dysfunction: a resting-state fMRI study. Andrology. (2017) 5:1073-81. doi: 10.1111/andr.12411

56. Schmidt C, Morris LS, Kvamme TL, Hall P, Birchard T, Voon V. Compulsive sexual behavior: prefrontal and limbic volume and interactions. Hum Brain Mapp. (2017) 38:1182-90. doi: 10.1002/hbm.23447

57. Kneer J, Borchardt V, Kärgel C, Sinke C, Massau C, Tenbergen G, et al. Diminished fronto-limbic functional connectivity in child sexual offenders. J Psychiatr Res. (2019) 108:48-56. doi: 10.1016/j.jpsychires.2018.01.012

58. Yin T, Liu Q, Ma Z, Li Z, Sun R, Ren F, et al. Associations between altered cerebral activity patterns and psychosocial disorders in patients with psychogenic erectile dysfunction: a mediation analysis of fMRI. Front Psychiatry. (2020) 11:583619. doi: 10.3389/fpsyt.2020.583619

59. Lu J, Yuan L, Jin J, Yang S, Zhang W, Li M, et al. Brain cortical complexity and subcortical morphometrics in lifelong premature ejaculation. Front Hum Neurosci. (2020) 14:283. doi: 10.3389/fnhum.2020.00283

60. Li B, Zhang L, Zhang Y, Chen Y, Peng J, Shao Y, et al. Decreased functional connectivity between the right precuneus and middle frontal gyrus is related to attentional decline following acute sleep deprivation. Front Neurosci. (2020) 14:530257. doi: 10.3389/fnins.2020.530257

61. Wang Y, Zhu W, Xiao M, Zhang Q, Zhao Y, Zhang H, et al. Hostile attribution bias mediates the relationship between structural variations in the left middle frontal gyrus and trait angry rumination. Front Psychol. (2018) 9:526. doi: 10.3389/fpsyg.2018.00526

62. Chang $\mathrm{CH}$, Grace AA. Inhibitory modulation of orbitofrontal cortex on medial prefrontal cortex-amygdala information flow. Cereb Cortex. (2018) 28:1-8. doi: 10.1093/cercor/ bhw342

63. Balasubramani PP, Pesce MC, Hayden BY. Activity in orbitofrontal neuronal ensembles reflects inhibitory control. Eur J Neurosci. (2020) 51:203351. doi: $10.1111 /$ ejn. 14638

64. Zhang B, Lu J, Xia J, Wang F, Li W, Chen F, et al. Functional insights into aberrant brain responses and integration in patients with lifelong premature ejaculation. Sci Rep. (2017) 7:460. doi: 10.1038/s41598-017-00421-3

65. Gao M, Feng N, Guo B, Wu J, Sun J, Zhang L, et al. Striatum-related Intrinsic connectivity deficits in lifelong premature ejaculation patients. Urology. (2020) 143:159-64. doi: 10.1016/j.urology.2020.06.001

66. Gao M, Feng N, Wu J, Sun J, Zhang L, Guo X, et al. Altered functional connectivity of hypothalamus in lifelong premature ejaculation patients. J Magn Reson Imaging. (2020) 52:778-84. doi: 10.1002/jmri. 27099

67. Matyi MA, Spielberg JM. Differential spatial patterns of structural connectivity of amygdala nuclei with orbitofrontal cortex. Hum Brain Mapp. (2021) 42:1391-405. doi: 10.1002/hbm.25300

68. Lu J, Zhang X, Wang H, Qing Z, Han P, Li M, et al. Short- and longrange synergism disorders in lifelong premature ejaculation evaluated using the functional connectivity density and network property. NeuroImage Clin. (2018) 19:607-15. doi: 10.1016/j.nicl.2018.05.025

69. Poeppl TB, Langguth B, Laird AR, Eickhoff SB. The functional neuroanatomy of male psychosexual and physiosexual arousal: a quantitative meta-analysis. Hum Brain Mapp. (2014) 35:1404-21. doi: 10.1002/hbm.22262

70. Briggs RG, Lin YH, Dadario NB, Kim SJ, Young IM, Bai MY, et al. Anatomy and white matter connections of the middle frontal gyrus. World Neurosurg. (2021) 150:e520-9. doi: 10.1016/j.wneu.2021.03.045

71. Hornberger $M$, Bertoux $M$. Right lateral prefrontal cortexspecificity for inhibition or strategy use? Brain. (2015) $138(\mathrm{Pt}$ 4):833-5. doi: 10.1093/brain/awv027

Conflict of Interest: The authors declare that the research was conducted in the absence of any commercial or financial relationships that could be construed as a potential conflict of interest.

Publisher's Note: All claims expressed in this article are solely those of the authors and do not necessarily represent those of their affiliated organizations, or those of the publisher, the editors and the reviewers. Any product that may be evaluated in this article, or claim that may be made by its manufacturer, is not guaranteed or endorsed by the publisher.

Copyright $\odot 2022$ Zhang, Yuan, Cui, Yuan and Jiang. This is an open-access article distributed under the terms of the Creative Commons Attribution License (CC BY). The use, distribution or reproduction in other forums is permitted, provided the original author(s) and the copyright owner(s) are credited and that the original publication in this journal is cited, in accordance with accepted academic practice. No use, distribution or reproduction is permitted which does not comply with these terms. 